\title{
Influenza virus hedges its bets
}

4

heterogeneity is a central feature of viral infection with major implications for infection outcomes
Extreme heterogeneity exists in both viral and host cell populations. For instance, influenza A viruses (IAVs) can replicate incomplete subsets of viral genes and exhibit substantial diversity in gene expression patterns between individual infected cells. However, little is known about how this heterogeneity influences infection dynamics and outcomes. Now, two recent studies shed light on the importance of heterogeneity in determining the outcome of IAV infections, either by shaping the immune response to infection, or by determining the potential for IAV to overcome species barriers.

In the first study, Sun, Vera et al. investigated the effects of viral population heterogeneity on the host response to IAV infection and found that single cell heterogeneity in viral gene expression can shape the innate antiviral response. First, using single cell RNA sequencing, the authors determined the viral and host transcriptional profiles in thousands of single cells infected with H1N1 or H3N2 IAVs. They observed enormous single cell heterogeneity in viral gene expression patterns in both total viral transcript levels and the levels of specific viral genes and found substantial differences in patterns of viral gene transcriptional heterogeneity at the single cell level between H1N1 and H3N2 IAVs.

Next, the authors investigated whether cell-to-cell heterogeneity in viral gene expression was associated with variation in the host gene transcriptome. Overall, they observed that infection significantly increased cell-to-cell heterogeneity in host gene transcription and found a relationship between viral gene expression and the host transcriptional response. Among the host genes that differed in expression between single cells were numerous genes that are crucial determinants of IAV infection outcome, including antiviral interferons and interferon-stimulated genes (ISGs). Interestingly, the authors found substantial differences in heterogeneity of expression of type III interferons and several ISGs in H1N1 (but not H3N2) infection.

At least half of all virions failed to express detectable levels of one or more viral genes. By comparing host gene expression in different populations of infected cells that differ in expression status of individual viral genes, the authors identified hundreds of genes that varied significantly according to the expression of specific viral genes. In particular, the absence of the non-structural (NS) gene segment was found to be a major determinant of interferon and ISG induction by the H3N2 IAV, but not the H1N1 IAV, suggesting that single cell heterogeneity in NS segment expression is an important determinant of the activation of the innate antiviral response.

In the second study, Phipps et al. investigated whether the incomplete viral genomes detected by Sun, Vera et al. create a reliance on multiple infection and whether complementation between incomplete genomes has a

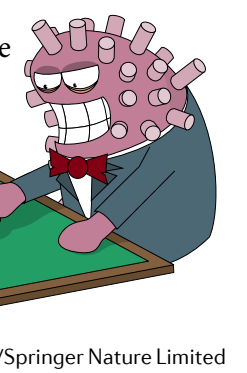

role in IAV overcoming species barriers. First, the authors used reassortment and co-infection as readouts to determine the extent to which IAV requires multiple infection for productive infection. By measuring co-infection and reassortment frequencies after single cycle infections of mammalian and avian cells with mammalian or avian IAVs, the authors observed that productive infection is often dependent on multiple infection and that the extent of this dependence varies with the virus strain and host species. Notably, the authors found that H9N2 avian IAVs are dependent on multiple infection in mammalian cells and that this dependence is reduced in avian cells. Infections of guinea pigs and quails confirmed the host-dependent reliance on multiple infection.

The reassortant analyses suggested that multiplicity of infection (MOI) enhances viral growth. The authors found that a higher MOI results in accelerated growth. Further experiments revealed that multiple infection augments viral RNA synthesis and that the viral RNA polymerase PA gene segment is the major determinant of multiple infection dependence for H9N2 viruses in mammalian systems.

In sum, these studies highlight that heterogeneity is a central feature of viral infection with major implications for infection outcomes.

Ashley York

ORIGINAL ARTICLES Sun, J. \& Vera, J. C. et al. Single cell heterogeneity in influenza A virus gene expression shapes the innate antiviral response to infection. PLoS Pathog. 16, e1008671 (2020)| Phipps, K. L. et al. Collective interactions augment influenza A virus replication in a host-dependent manner. Nat. Microbiol. https://doi.org/10.1038/ 541564-020-0749-2 (2020) 